\title{
PLANTAS EMERGENTES Y FLOTANTES EN LA DIETA DEL MANATÍ (FAMILIA: TRICHECHIDAE: TRICHECHUS MANATUS) EN EL CARIBE DE COSTA RICA
}

\author{
Alexander Gómez Lépiz ${ }^{1}$
}

\begin{abstract}
RESUMEN
Para determinar la dieta del manatí y cuantificar el uso y la disponibilidad de las plantas contenidas en su dieta, se realizaron recorridos sobre diferentes sectores del Parque Nacional Tortuguero, en la búsqueda de rastros de alimentación dejados por los manatíes. En los sitios de ramoneo recientes, la proporción de plantas se cuantificó a través de un análisis con los porcentajes de las plantas encontradas en los sitios de alimentación (uso) y sobre las áreas muestreadas alrededor de estos (disponibilidad). Se pudo determinar que dos especies de pastos: gamalote (Paspalum repens) y pará (Urochloa mutica), son las plantas que principalmente selecciona el manatí para su consumo. La presente investigación ha permitido cuantificar la forma en que el manatí consume los recursos alimenticios en la zona. Esta ha sido la base para otras investigaciones que requieren de información sobre los elementos de la dieta para el cebado como técnica de captura, también se están implementando propuestas de sitios sensibles para la conservación de manatíes con base en la distribución de las plantas que este animal consume en la zona.
\end{abstract}

Palabras claves: Análisis nutricional, Costa Rica, dieta, Trichechus manatus, Parque Nacional Tortuguero.

\begin{abstract}
Trips were conducted on different sectors of the Tortuguero National Park in search of traces of food left by the manatees to determine their diet and quantify the use and availability of plants contained in their diet,. In recent browsing sites the proportion of plants was quantified by analyzing the percentages of the plants found in the feeding sites (use) and sampled areas around them (availability). It was determined that two grass species: gamalote (Paspalum repens) and (Urochloa mutica) are plants that the manatees primarily selected for consumption. The present study permitted to determine how the manatee consumes food resources in the area. This has been the basis for other research requiring information on dietary factors. There are also other proposals being implemented for baiting and capture techniques in sensitive sites for the conservation of manatees, based on the distribution of the plants that the animals consume in the area.
\end{abstract}

Keywords: Nutritional analysis, Costa Rica, diet, Trichechus manatus, Tortuguero National Park.

\section{INTRODUCCIÓN}

La mayoría de los estudios sobre preferencia identifican los recursos usados por los animales y documentan la disponibilidad de estos, para luego determinar cuáles son seleccionados con mayor frecuencia sobre otros (Thomas et al. 2001). Los patrones de comportamiento alimenticio de los herbívoros en general indican que ellos incrementan el consumo de alimentos que les brindan un mayor rendimiento nutricional, y limitan el consumo de plantas tóxicas o de baja calidad (Launchbaugh et al. 2001).

El manatí del Caribe, Trichechus manatus, pertenece al orden Sirenia, un grupo formado solo por mamíferos herbívo-

1 Instituto Internacional en Conservación y Manejo de Vida Silvestre, Universidad Nacional, Apartado 1350-300, Heredia, Costa Rica.gmanatusl@gmail.com 
ros acuáticos (Burn, 1986). Los manatíes poseen un sistema digestivo con altos niveles de eficiencia, pero para satisfacer sus requerimientos metabólicos necesitan consumir grandes cantidades de vegetación acuática, la cual posee alto porcentaje de fibra cruda y cantidades bajas de proteína (Smith, 1993).

Algunas investigaciones abordan aspectos de la dieta de los manatíes y la forma en cómo estos utilizan los recursos alimenticios (Burn, 1986; Provancha y Hall, 1991; Colares y Colares, 2002). Esas investigaciones, en su mayoría, relacionan los procesos metabólicos del sistema digestivo con las diferentes especies de plantas que conforman su dieta.

Sin embargo, son escasos los estudios sobre la dieta del manatí que intentan explicar la forma en que este escoge los diferentes elementos que conforman su dieta (Colares y Colares, 2002). Otras investigaciones abordan la relación entre las plantas que consumen los manatíes y la presencia de estas en la dieta de la especie, mediante procesos metabólicos (Burn, 1986). Los estudios que abarcan con mayor detalle la dieta de la especie y sobre aspectos de preferencia que esta hace de los recursos alimenticios, se han realizado para las poblaciones de manatíes de Florida (Estados Unidos), por ejemplo, Provancha y Hall (1991). Las variaciones en las características del hábitat para esta especie entre regiones (Reynolds y Ode11, 1991; Reep y Bonde, 2006; Guterres et al. 2008), limitan la aplicación de esos estudios a las condiciones en que viven los manatíes en Costa Rica y otras áreas tropicales con condiciones ecológicas similares (Guterres et al. 2008).

La investigación acerca de los manatíes en Costa Rica es escasa, y la información relacionada con la dieta de esta especie está orientada hacia observaciones sobre sitios de alimentación y listados de especies que la conforman (Jiménez, 2003), sin profundizar en la composición y la trascendencia de las plantas que conforman la dieta de este mamífero.

Los objetivos del presente estudio fueron: i) identificar la disponibilidad de las especies de plantas emergentes y flotantes que conforman la dieta del manatí y relacionarlas con el uso, ii) explicar la selección de plantas emergentes y flotantes a partir de sus características nutricionales.

\section{ÁREA DE ESTUDIO}

El área de estudio se ubica en el Parque Nacional Tortuguero (PNT). Este parque nacional se localiza en la costa Caribe de la Provincia de Limón, a $80 \mathrm{~km}$ al noreste del cantón central de esta provincia. La zona es considerada como bosque tropical muy húmedo (Barrantes et al. 1985), con una precipitación que oscila en promedio entre $4500 \mathrm{~mm}$ y $5500 \mathrm{~mm}$, con la mínima precipitación en marzo (250 mm aproximadamente) y en noviembre la máxima (630 $\mathrm{mm}$ aproximadamente), y la temperatura promedio anual oscila entre $\operatorname{los} 25^{\circ} \mathrm{C}-30^{\circ} \mathrm{C}$ (IMN, 1975-1995).

A pesar de la abundante vegetación sobre las orillas de los canales (palmas, árboles, arbustos), la baja visibilidad del agua de los humedales locales convierte a estos en lugares pobres en pastos sumergidos, presentando en cambio abundantes bancos de vegetación flotante, emergente o de orilla (Jiménez, 1998).

\section{MATERIALES Y MÉTODOS}

Para esta investigación no se contó con la posibilidad de observar manatíes, por lo que se consideró la presencia de 
sus rastros sobre las plantas consumidas, como método indirecto para identificar su dieta. Los manatíes en el área de estudio son sensibles a cualquier perturbación, y huyen con facilidad ante situaciones de amenaza, lo que dificulta la observación directa de estos. Ante tal situación, se utilizaron los rastros dejados por manatíes para desarrollar el presente estudio sobre hábitos alimenticios, según lo definido por Aranda (2000) para la observación indirecta de mamíferos como opción de desarrollo de estudios sistemáticos.

Para definir la dieta de los manatíes en el PNT se utilizaron solo especies de plantas acuáticas emergentes y flotantes. Las visitas al área de estudio se hicieron entre los meses de febrero del 2005 y marzo del 2006, con una duración de dos a tres semanas por mes. En total se realizaron 53 recorridos en 6 diferentes sectores en que se dividió el parque nacional y cada sector fue visitado entre 8 y 10 ocasiones.

La condición principal para realizar la medida sobre un sitio de ramoneo fue que el rastro debía ser fresco (no más de una noche). Los recorridos en busca de rastros iniciaban entre las 05:15 y 05:45 de la mañana, según la claridad de la época del año. La búsqueda de los rastros se realizó en un bote, a una velocidad de $3 \mathrm{~km}$ por hora y se revisaban ambas márgenes del sector del parque nacional recorrido durante el muestreo.

$\mathrm{Se}$ recolectaron muestras de aquellas plantas (Paspalum repens, Urochloa mutica, Eichhornia crassipes, Ludwigia helminthorrhiza y Hydrocotyle ranunculoides) que fueron consumidas en los sitios de alimentación para su posterior identificación y análisis nutricional. La identificación de las plantas y el análisis nutricional se realizaron en los laboratorios de Botánica y Análisis de Productos Animales y Vegetales de la Universidad
Nacional en Heredia, Costa Rica, respectivamente.

\section{Determinación de la disponibilidad y el uso de recursos alimenticios mediante el método de armazón de puntos}

Durante los recorridos se consideró en cada sitio de alimentación lo siguiente: a) se estimó la abundancia de las plantas flotantes $\mathrm{y}$ emergentes en los sitios donde se alimentaron los manatíes como una medida de uso, b) se midió de igual forma la abundancia de plantas en las áreas cercanas a esos sitios de alimentación como una medida para estimar la disponibilidad. Se utilizó el método de "armazón de puntos" (Litvaitis et al. 1994), adaptado para este estudio según la estructura presentada en la figura 1 .

En las áreas donde el manatí se alimentó, las observaciones se llevaron a cabo de la siguiente manera: en los sitios de alimentación (como medida de uso): a) las puntas de las varillas se dejaban caer sobre el sitio de alimentación y se registraba solamente la especie de planta que tocaba cada punta de cada varilla; b) cuando el rastro era mayor al tamaño de la estructura, se levantaban las varillas y se colocaban de nuevo hasta cubrir totalmente el área donde el manatí se había alimentado. Se procedió de la misma forma para realizar una medida al lado del área de comido $\mathrm{y}$ tres medidas a cada lado del área de comido con una distancia de $25 \mathrm{~m}$ (Fig. 2).

\section{Pruebas estadísticas}

La deficiencia en algunos supuestos imposibilitó el desarrollo de análisis paramétricos, por lo tanto, el análisis estadístico se desarrolló como sigue: fue utilizado el programa HABUSE, que basa su análisis en intervalos de Bonferroni. Dicho programa determina, mediante una prueba de Bondad de Ajuste de Chi-cuadrado, las diferencias 


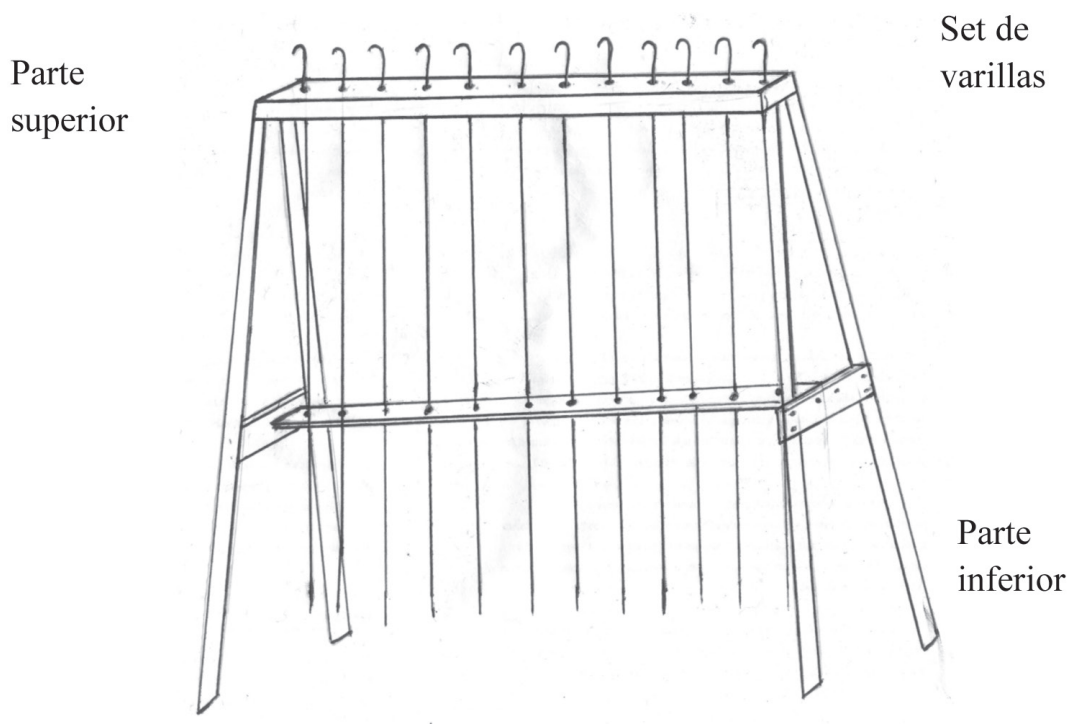

Fig. 1. Estructura de armazón de puntos para la caracterización y cuantificación de las plantas observadas sobre las áreas donde los manatíes se alimentaban y alrededor de estas. Las puntas de las varillas fueron utilizadas para la observación de las plantas consumidas

Fig. 1. Points structure for the characterization and quantification of the plants observed in areas where the manatees ate and in the surroundings. The tips of the rods were used for observation of the plants consumed

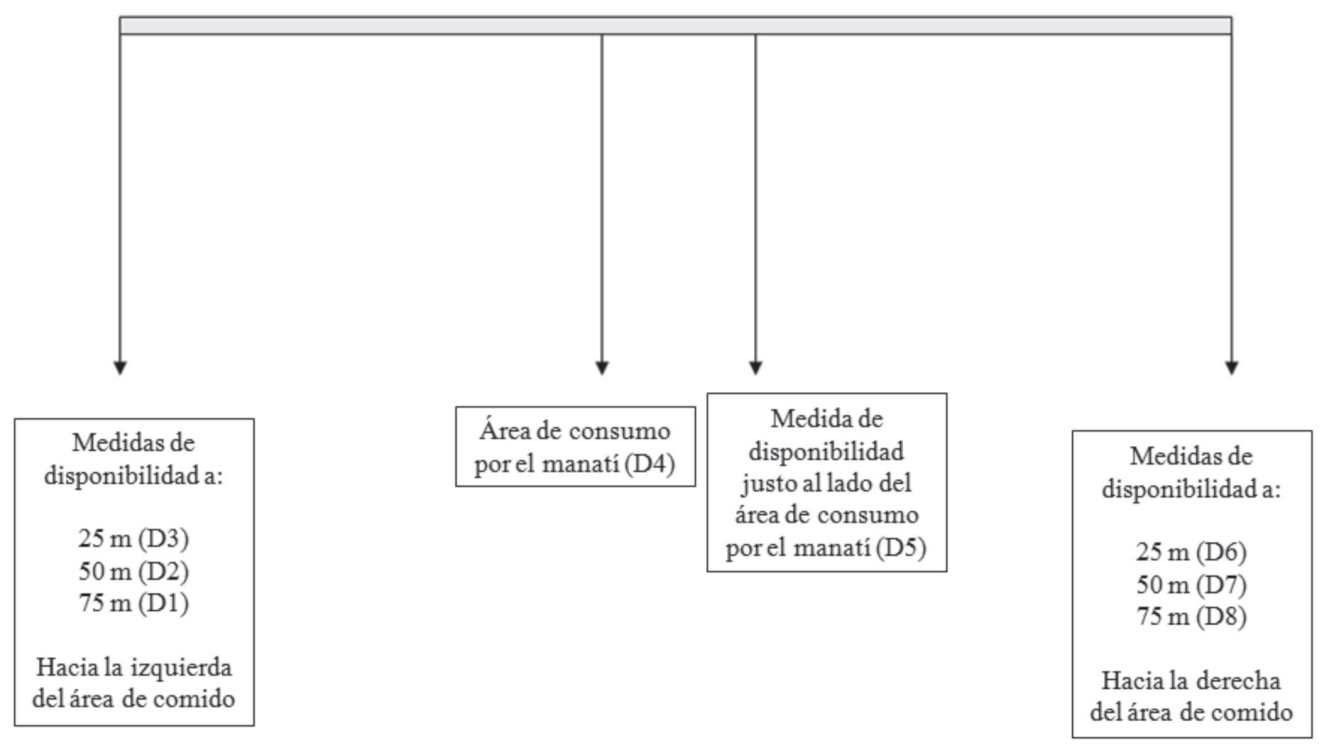

Fig. 2. Esquema de medición sobre el área de consumo por el manatí y sitios alrededor

Fig. 2. Diagram of measures in the manatees feeding area and in the surrounding areas 
Cuadro 1. Plantas encontradas sobre las áreas donde los manatíes se alimentaron y alrededor de estas

Table 1. Plants found over the areas where manatees ate and areas around the same

\begin{tabular}{llllc}
\hline \multicolumn{1}{c}{ Familia } & \multicolumn{1}{c}{ Nombre científico } & \multicolumn{1}{c}{$\begin{array}{c}\text { Nombre } \\
\text { común }\end{array}$} & $\begin{array}{c}\text { Condición de } \\
\text { consumo }\end{array}$ & $\begin{array}{c}\text { Clasificación de } \\
\text { la planta }\end{array}$ \\
\hline Poaceae & Paspalum repens & gamalote & Consumida & Emergente \\
Poaceae & Urochloa mutica & pará & Consumida & Emergente \\
Poaceae & Oriza latifolia & zacate rojo & Consumida & Emergente \\
Poaceae & Panicum maximum & zacate navaja & Consumida & Emergente \\
Pontederiaceae & Eichhornia crassipes & choreja & Consumida & Flotante \\
Apiaceae & Hydrocotyle ranunculoides & comalillo & Consumida & Flotante \\
Onagraceae & Ludwigia helminthorrhiza & lirio rosa & Consumida & Flotante \\
Pontederiaceae & Eichhornia azurea & lirio de agua & No consumida & Flotante \\
Onagraceae & Ludwigia spp & hierba de agua & No consumida & Flotante \\
\hline
\end{tabular}

significativas entre la utilización esperada de los tipos de vegetación (basada en la disponibilidad) y las observaciones de uso.

Cuando se detectan diferencias significativas entre la utilización y la disponibilidad de los tipos de vegetación, los intervalos de Bonferroni son utilizados para determinar los tipos de recursos seleccionados. Byers y Steinhorst (1984) mencionan detalles sobre algoritmos y supuestos de este procedimiento. Con este análisis fue determinada la condición final de los recursos alimenticios en la dieta del manatí para el área de estudio.

Para la prueba de análisis de interacción se utilizó el programa Statgraphics Centurium XV (Manugistics, 2005), a través del cual se realizaron pruebas de Análisis de Varianza Multifactorial, en el que se compararon las medias de los porcentajes observados para las especies de plantas (tratamientos) sobre los sitios alrededor (factores) de las áreas de alimentación, frente a los mismos datos obtenidos sobre aquellos sitios donde el manatí se alimentó. Posteriormente, se utilizaron intervalos de
Scheffé, para así diferenciar las plantas con medias mayores en las áreas de consumo.

Los valores de intervalos de Bonferroni se obtuvieron de los sitios para medidas de disponibilidad, al lado (D5) y el extremo hacia la derecha (D8) e izquierda (D1), según la figura 2 para el área de comido y alrededores.

Para determinar la condición final de uso de las plantas, se consideró el valor real de uso sobre el área de comido y se comparó con los valores dados para los intervalos de Bonferroni basados en las medidas de disponibilidad. La valoración de la condición final de uso se presentó como sigue: (-) los valores de uso esperado según la disponibilidad fueron menores que los valores de uso real; $(+)$ los valores de uso esperado según la disponibilidad fueron mayores que los valores de uso real; (=) no se detectó diferencia entre los valores de uso esperado según la disponibilidad y los valores de uso real (Cuadro 2).

\section{Análisis nutricional}

Se recolectaron muestras de las principales plantas que conformaron la dieta de los 
Cuadro 2. Condición de uso/disponibilidad de las especies de plantas encontradas sobre las áreas de consumo de los manatíes y sitios evaluados alrededor de estas Table 2. Use/availability condition of the plants found where the manatees ate and in the surrounding sites evaluated

\begin{tabular}{|c|c|c|c|c|c|c|c|}
\hline \multirow[b]{2}{*}{ Planta } & \multirow{2}{*}{$\begin{array}{l}\text { Valor real } \\
\text { de uso } \\
\text { Área de } \\
\text { comido }\end{array}$} & \multicolumn{3}{|c|}{$\begin{array}{c}\text { Valores esperados de uso según los valores de } \\
\text { dispon ibilidad } \\
\text { (Intervalos de Bonferroni*) }\end{array}$} & \multicolumn{3}{|c|}{$\begin{array}{l}\text { Condición } \\
\text { final de uso }\end{array}$} \\
\hline & & D1 & D5 & D8 & D1 & D5 & D8 \\
\hline $\begin{array}{l}\text { Paspalum repens } \\
\text { (gamalote) }\end{array}$ & 0.421 & $0.108-0.136$ & $0.306-0.344$ & $0.186-0.219$ & - & - & - \\
\hline $\begin{array}{l}\text { Urochloa mutica } \\
\text { (pará) }\end{array}$ & 0.346 & $0.181-0.214$ & $0.330-0.369$ & $0.184-0.218$ & - & $=$ & - \\
\hline $\begin{array}{l}\text { Hydrocotyle } \\
\text { ranunculoides } \\
\text { (comalillo) }\end{array}$ & 0.188 & $0.352-0.392$ & $0.210-0.244$ & $0.339-0.379$ & + & + & + \\
\hline $\begin{array}{l}\text { Eichhornia } \\
\text { crassipes (choreja) }\end{array}$ & 0.032 & $0.080-0.104$ & $0.048-0.066$ & $0.083-0.108$ & + & + & + \\
\hline $\begin{array}{l}\text { Panicum maximum } \\
\text { (zacate navaja) }\end{array}$ & 0.010 & $0.018-0.031$ & / & $0.002-0.009$ & + & l & - \\
\hline $\begin{array}{l}\text { Ludwigia } \\
\text { helminthorrhiza } \\
\text { (lirio rosa) }\end{array}$ & 0.000 & $0.133-0.163$ & $0.024-0.038$ & $0.111-0.139$ & + & + & + \\
\hline $\begin{array}{l}\text { Oriza latifolia } \\
\text { (zacate rojo) }\end{array}$ & 0.002 & $0.007-0.015$ & l & $0.005-0.012$ & + & / & + \\
\hline $\begin{array}{l}\text { Ludwigia spp } \\
\text { (hierba de agua) }\end{array}$ & 0.000 & / & $0.006-0.015$ & I & + & / & / \\
\hline $\begin{array}{l}\text { Eichhornia azurea } \\
\text { (lirio de agua) }\end{array}$ & 0.000 & $0.002-0.009$ & / & $0.001-0.005$ & + & l & + \\
\hline
\end{tabular}

* Intervalos de Bonferroni $P<0.05$.

Simbología:

D5, D8, D1: medidas de disponibilidad al lado y el extremo hacia la derecha e izquierda del área de comido, respectivamente.

D5, D8, D1: availability measures next to the eating area and right and left to this site respectively.

Condición final de preferencia: (-) los valores de uso esperado según la disponibilidad fueron menores que los valores de uso real; $(+)$ los valores de uso esperado según la disponibilidad fueron mayores que los valores de uso real; $(=)$ no se detectó diferencia entre los valores de uso esperado según la disponibilidad y los valores de uso real; (/) no se registraron datos.

Final condition of preference. (-) expected use values according to availability were lower than actual use values; $(+)$ expected use values according to availability were greater than actual use values; $(=)$ no difference detected between the values of expected use based on availability and actual use values, (/) no data.

manatíes, para intentar explicar, mediante las características nutricionales, la relevancia de estas en dicha dieta. Se utilizaron dos especies de pasto, gamalote (P. repens) y pará ( $U$. mutica) y 3 especies de lirios acuáticos, choreja (E. crassipes), lirio rosa (L. helminthorrhiza) y comalillo (H. ranunculoides).
Para las muestras recolectadas para el análisis se consideraron las partes de la planta que los manatíes consumían (hoja y/o ta1lo). Todas las muestras fueron recolectadas en las primeras horas de la mañana, entre las 06:00 y 08:00; el peso de las muestras fue de 900 g por cada planta, se recolectaron dos 
muestras por cada una de las plantas antes mencionadas y estas muestras fueron colocadas en hielo para su traslado hasta el laboratorio, donde se les realizaron pruebas para determinar los porcentajes de proteína cruda (PC\%), fibra neutro detergente (FND\%) y fibra ácido detergente (FAD\%). El procedimiento que se siguió para evaluar estos parámetros fue el método de AOAC (1984).

A las muestras de $P$. repens y $U$. mutica se les realizó un análisis para determinar el contenido de lignina y de celulosa, con el fin de evaluar si estos componentes explicaban la diferencia en las medias de estas especies con respecto a las otras plantas encontradas. El resto de especies que conforman la dieta del manatí para este último análisis se descartaron, debido a sus bajos contenidos de fibra en relación con $P$. repens y $U$. mutica, y además por ser estas últimas las especies que mayoritariamente consumió el manatí.

Mediante una prueba de Bondad de Ajuste y el desarrollo de intervalos de Bonferroni se diferenciaron a las especies según los valores obtenidos para los contenidos de fibra y proteína. Para ambas pruebas se siguió el procedimiento estadístico que describen Sokal y Rohlf (1979).

\section{RESULTADOS}

\section{Dieta de la especie}

Se encontraron 57 sitios que fueron ramoneados por los manatíes, en estos se observaron 7 especies consumidas en más de una ocasión y solo dos plantas localizadas en esos sitios no mostraron evidencia de ramoneo. La familia Poaceae presentó la mayor cantidad de especies en la dieta del manatí y el resto de familias (Pontederiaceae, Apiaceae, Onagraceae) estuvieron representadas por una sola es- pecie (Cuadro 1). Según la clasificación propuesta por Guterres et al. (2008), las plantas consumidas fueron emergentes en su mayoría (4 especies) y un número similar (3 especies) fueron flotantes, no hubo evidencia de consumo de plantas sumergidas, arbóreas ni anfibias.

\section{Preferencia de recursos alimentarios Análisis de uso/disponibilidad}

El cuadro 1 presenta las plantas encontradas sobre las áreas donde el manatí se alimentó y los sitos alrededor de esas áreas. La información obtenida indica que: dos especies de plantas solo fueron observadas en los alrededores de las áreas de comido y nunca fueron consumidas por los manatíes: hierba de agua (Ludwigia spp) y lirio de agua (Eichhornia azurea), y la frecuencia de aparición de estas fue baja.

Otras especies, como el zacate navaja (Panicum maximum) y el zacate rojo (Oriza latifolia), fueron consumidas por el manatí y al igual que las especies anteriores, la aparición de estas sobre aquellos sitios donde se alimentó también fue baja (menor al 1.5\%).

L. helminthorrhiza fue otra planta que se observó en pocas ocasiones alrededor de las áreas donde el manatí se alimentaba y fue la especie que se encontró con menor frecuencia sobre los sitios de alimentación propiamente $(0.33 \%)$, al igual que $E$. crassipes $(3.55 \%)$. H. ranunculoides es una de las tres especies que aparece con mayor frecuencia sobre los sitios donde el manatí se alimentó (18.17\%); sin embargo, estas especies mostraron valores de uso esperado, según la disponibilidad, mayores que los valores de uso real, lo que podría indicar que los manatíes tienden a evitar sitios con presencia de estas plantas (Fig. 3).

Por el contrario, se observó que $P$. repens y $U$. mutica $(41 \%$ y $35.22 \%$, res- 


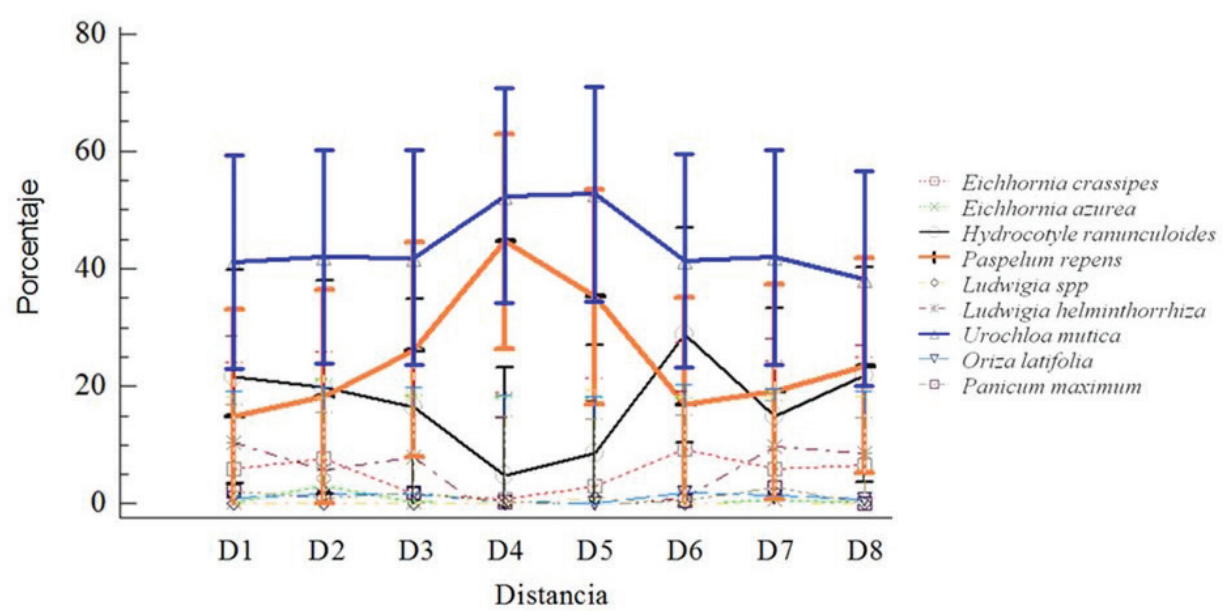

Fig. 3. Relación entre los porcentajes de observación de las especies de plantas sobre las áreas donde los manatíes se alimentaron y lugares alrededor de estas

Fig. 3. Relationship between the observation percentage of the plant species and the areas where the manatees are fed and the places around them

Distancias (D): D1, D2, D3 = lugares a la izquierda del área donde los manatíes comieron, D6, D7, D8 = lugares a la derecha del área donde los manatíes comieron, D4 = área donde los manatíes se alimentaron, D5 = lugar al lado del área donde los manatíes se alimentaron.

Distances (D): D1, D2, D3 = places to the left of the area where manatees ate, D6, D7, D8 = places to the right of the area where manatees ate, D4 $=$ area where manatees ate, D5 = place adjacent to the areas where manatees ate.

pectivamente) fueron las dos especies con mayor ocurrencia sobre sitios de comido, ya que estas plantas fueron las únicas que sus valores de uso esperado, según la disponibilidad, fueron menores que los valores de uso real (Cuadro 2). Esto evidencia un patrón de búsqueda de los manatíes hacia sitios para alimentarse donde se encuentran estos pastos en cantidades considerables, ese mismo patrón lo reafirma el análisis de interacción descrito a continuación.

\section{Análisis de interacción}

Mediante el análisis de interacción en la figura 1 , se hace más evidente la diferencia entre los pastos, $P$. repens y $U$. mutica, en relación con el resto de las especies encontradas en la dieta del manatí $(F=80.75, g . l$
$=8, P<0.001)$. Estas especies fueron seleccionadas por los manatíes en las áreas de vegetación comida, con mayor frecuencia que las demás especies de su dieta $(F$ $=15.03, g . l=56, P<0.001)$. En la figura 1 , se observa cómo estas dos especies, conforme se encontraron más cerca de los sitios escogidos por el manatí para alimentarse, los valores de estas aumentan. Esta situación es contraria a lo que sucede con H. ranunculoides, que tiende a disminuir sus valores al acercarse a los sitios donde se alimentó el manatí, inclusive, se puede notar cómo en ese punto, la presencia de la especie muestra los porcentajes más bajos registrados en este estudio.

Las otras plantas podrían ser consideradas en términos de consumo como un grupo de poca relevancia en la dieta del 
manatí, ya que los valores encontrados de estas especies son bajos, inclusive sobre las áreas de muestreo vecinas a los sitios de alimentación. En este grupo (resto de especies) no se observó ninguna tendencia en el análisis relacionado con los lugares de vegetación consumida por el manatí.

\section{Características nutricionales de los principales recursos seleccionados}

Los valores en porcentaje de las plantas emergentes y flotantes que constituyeron la dieta de los manatíes, según su composición química para los componentes considerados para el análisis, están representados en el cuadro 3. Los valores en porcentaje de materia seca encontrados para los pastos analizados $(U$. mutica y $P$. repens) fueron mayores que para el resto de las especies $(H$. ranunculoides, E. crassipes y L. helminthorrhiza). Los pastos presentaron casi el doble del contenido de materia seca que las otras especies consideradas.

En cuanto a la proteína cruda, los valores más altos fueron encontrados en dos especies de lirios: $H$. ranunculoides y L. helminthorrhiza; por otro lado, dos especies mostraron valores similares en su contenido de proteína, $U$. mutica y $E$. crassipes. Sin embargo, $P$. repens presentó los valores más bajos en contenido de proteína cruda, alcanzando apenas un poco más de la mitad del valor encontrado para la especie con el mayor contenido proteico (H. ranunculoides).

Los porcentajes de fibra neutro detergente fueron mayores en tres especies, los dos pastos (U. mutica y $P$. repens) y una especie de lirio (E. crassipes), las otras dos especies (H. ranunculoides y $L$. helminthorrhiza) presentaron los valores más bajos para este parámetro.
La evaluación relacionada con los parámetros de celulosa y lignina para ambas especies de pastos consideradas en este análisis indica, que los porcentajes obtenidos para ambas son parecidos, aunque $P$. repens presenta los mayores valores tanto en el porcentaje de celulosa como de lignina.

\section{DISCUSIÓN}

\section{Dieta de la especie}

La dieta de los manatíes en el PNT consiste de: P. repens, U. mutica, O. latifolia, $P$. maximum, E. crassipes, $H$. ranunculoides y L. helminthorrhiza. Reynolds et al. (1995), Jiménez (1999), Smethurst y Nietschmann (1999) y Jiménez (2003), coinciden con algunas de estas especies, pero no reconocen la importancia de una especie o grupo de especies sobre otras.

La mayoría de las plantas que reportan estos autores son emergentes $\mathrm{y}$ flotantes y la planta que se reporta con mayor frecuencia es E. crassipes, que es considerada la especie más importante en la dieta de los manatíes, junto a los pastos acuáticos en la zona (Jiménez, 1999). Históricamente, E. crassipes fue una planta abundante dentro del área de estudio y su presencia se ha reducido con el tiempo y se ha visto una aparente disminución marcada durante el período en que se desarrolló la presente investigación (J. Martínez, comunicación personal, 5 de agosto del 2005; B. Sambola, comunicación personal, 5 de agosto del 2005).

La aparente disminución de esta planta en la zona plantea la necesidad de realizar estudios más específicos sobre la dieta del manatí y se perfilan algunas ideas para explicar el fenómeno ocurrido con esta planta, como la presencia de herbicidas usados en 
cultivos como la piña (IRET, 2007); por otro lado, la explicación podría concentrarse en fenómenos naturales propios de la composición y dinámica del agua.

En comparación con otros estudios, podría decirse que la cantidad de plantas reportadas para la dieta del manatí es baja, según lo observado en otras investigaciones en ecosistemas similares a los humedales del PNT (Colares y Colares, 2002). La cantidad de elementos presentes en la dieta del manatí podrían aumentar si se utilizaran técnicas que brinden mayor detalle, como la obtención de heces o análisis del tracto digestivo de los animales (Mignucci-Giannoni, 1998). La metodología que se utilizó limita la investigación de aquellas especies que son representativas en los sitios de alimentación, ante la dificultad para obtener información directa. Especies como Pistia sp, una planta de tamaño pequeño, podría ser consumida de forma indirecta, ya que en general se encuentra mezclada con otras plantas que el manatí busca directamente para alimentarse, como U. mutica y P. repens.

\section{Preferencia de recursos alimenticios sobre las áreas de comido}

La teoría de selección de recursos señala que los recursos de preferencia son aquellos que se usan desproporcionalmente a su disponibilidad (Manly et al. 2002). De las especies reportadas en la dieta del manatí, durante este estudio, se encontró claramente un uso desproporcional hacia dos pastos acuáticos de la zona: U. mutica y $P$. repens (Cuadro 2).

Estas mismas especies fueron las plantas que los manatíes consumieron mayoritariamente en los sitios donde se encontraron rastros de alimentación (Fig.1). Ambos resultados apuntan a que estas dos especies podrían conside- rarse como los recursos alimenticios de mayor relevancia para el manatí dentro del área de estudio, debido a la cantidad de registros obtenidos en los lugares donde la vegetación fue consumida por estos animales.

En apariencia, se pueden reconocer ciertas tendencias de las plantas encontradas tanto en los sitios donde se midió la disponibilidad como en aquellos considerados para evaluar el uso (Fig. 1). Según la descripción realizada por Johnson (1980), $U$. mutica y $P$. repens son las especies preferidas, en tanto que el comalillo tiende a un patrón de especie evitada, ya que el uso fue notoriamente menor que su disponibilidad. En otro grupo de especies no fue posible reconocer ningún patrón, por lo cual podrían considerarse a estas especies como indiferentes en la dieta, debido a la tendencia de estas en el análisis y a la relación de consumo con respecto a lo observado con el resto de las plantas consideradas como parte de la dieta. A pesar de esto, en este estudio no se analizó la importancia de esas especies en la dieta del manatí. Smith (1993) plantea que los manatíes también usan recursos alimenticios alternativos, cuando el material de forrajeo que comúnmente utilizan se reduce, o bien, cuando se necesita algún suplemento en la dieta de la especie, por lo que no se debe subestimar la presencia de esas plantas en la dieta (evitadas e indiferentes).

\section{Análisis nutricional}

Las características nutricionales consideradas para el análisis (Cuadro 3), en el estudio, marcan una tendencia en relación con las plantas que conformaron la dieta del manatí. Los valores encontrados para los parámetros analizados dividen la dieta de esta especie en dos grupos: los pastos y los lirios (Fig. 4). 
Cuadro 3. Composición química de las principales especies de plantas emergentes y flotantes que conformaron la dieta de los manatíes dentro del Parque Nacional Tortuguero Table 3. Chemical composition of the main species of emergent and floating plants that conformed the diet of manatees in Tortuguero National Park

\begin{tabular}{lccccccc}
\hline \multicolumn{1}{c}{ Planta } & $\begin{array}{c}\text { Materia } \\
\text { seca } \mathbf{6 0} \\
\mathbf{( \% )}\end{array}$ & $\begin{array}{c}\text { Humedad } \\
\mathbf{( \% )}\end{array}$ & $\begin{array}{c}\text { Proteína } \\
\mathbf{c r u d a} \\
\mathbf{( \% )}\end{array}$ & $\begin{array}{c}\text { Fibra neutro } \\
\text { detergente } \\
\mathbf{( \% )}\end{array}$ & $\begin{array}{c}\text { Fibra ácido } \\
\text { detergente } \\
\mathbf{( \% )}\end{array}$ & $\begin{array}{c}\text { Celulosa } \\
\mathbf{( \% )}\end{array}$ & $\begin{array}{c}\text { Lignina } \\
\mathbf{( \% )}\end{array}$ \\
\hline $\begin{array}{l}\text { Paspalum repens } \\
\text { (gamalote) }\end{array}$ & 13.11 & 86.89 & 14.37 & 60.93 & 32.92 & 37.38 & 8.68 \\
$\begin{array}{l}\text { Urochloa mutica } \\
\text { (pará) }\end{array}$ & 11.73 & 88.27 & 19.17 & 63.87 & 32.51 & 33.34 & 6.12 \\
$\begin{array}{l}\text { Hydrocotyle ranunculoides } \\
\text { (comalillo) }\end{array}$ & 6.32 & 93.68 & 27.91 & 32.21 & 19.28 & N.E. & N.E. \\
$\begin{array}{l}\text { Eichhornia crassipes } \\
\text { (choreja) }\end{array}$ & 6.24 & 93.76 & 19.85 & 55.75 & 24.27 & N.E. & N.E. \\
$\begin{array}{l}\text { Ludwigia helminthorrhiza } \\
\text { (lirio rosa) }\end{array}$ & 7.00 & 93.00 & 25.04 & 26.11 & 17.28 & N.E. & N.E. \\
\hline
\end{tabular}

N.E. $=$ no evaluado. N.E. $=$ not evaluated.

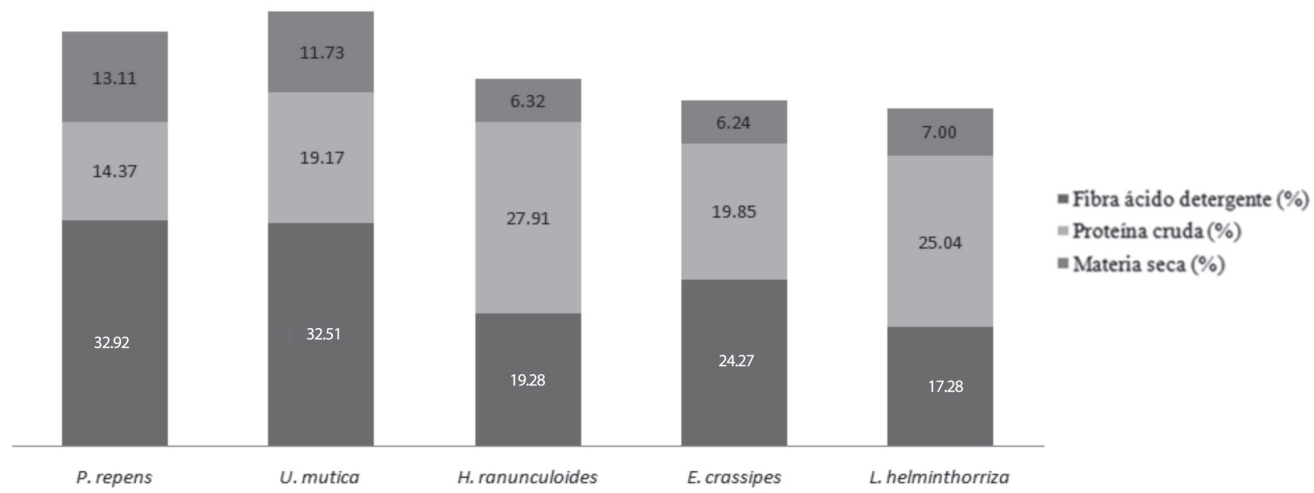

Planta

Fig. 4. Porcentajes de componentes nutricionales de las plantas observadas en la dieta del manatí

Fig. 4. Percentages of nutritional components of the plants observed in the diet of the manatee

Los bajos contenidos de fibra encontrados en las especies de lirios definirían la condición de esas plantas en la dieta del manatí como evitadas o indiferentes, según se observó durante este estudio. Nutricionalmente, estas plantas podrían ser diferentes a los requerimientos de un sistema digestivo adaptado sobre todo a la extracción de energía a partir de la cantidad de fibra contenida en las plantas
(Smith, 1993). Sin embargo, el consumo de estas plantas podría constituir un componente alternativo de la dieta, como es característico en muchos herbívoros.

Según el análisis desarrollado, los pastos $U$. mutica y $P$. repens y el lirio $E$. crassipes se pueden considerar en un grupo que comparten altos valores de fibra y bajos contenidos de proteína. Esto explica que ambos pastos conforman los dos com- 
ponentes principales en la dieta de los manatíes. E. crassipes, por el contrario, en la actualidad es una especie escasa en la zona (Obs. Pers.), lo cual explicaría los pocos registros de esta planta en la dieta del manatí, si se consideran investigaciones anteriores donde esta aparece como un componente fundamental en la alimentación de esta especie (Jiménez, 2003).

Una explicación para la situación antes planteada, es que la estructura en la composición vegetal de las plantas acuáticas podría verse afectada (positiva o negativamente) por la presencia de algunas sustancias en el agua, provenientes de monocultivos que rodean el ecosistema (IRET, 2007). Este punto debería ser motivo de estudio de posteriores investigaciones donde se evalúen aspectos ecológicos para esta especie, ya que las plantas acuáticas constituyen un aspecto fundamental para la sobrevivencia del manatí.

Por el contrario, los lirios, H. ranunculoides y L. helminthorrhiza, presentan contenidos altos en proteína y bajos en fibra. Esta situación podría explicar la frecuencia con que estas especies fueron consumidas. El consumo de estas plantas podría representar una condición alternativa en la dieta del manatí para suplir algunos componentes que las plantas consumidas con mayor frecuencia no brindan, como algunos micronutrientes o minerales, como se ha estudiado con detalle en la dieta para otros herbívoros como los pecaríes (Tayassu pecari) (Altrichter, 1997; López et al. 2006).

Otro aspecto por considerar, desde el punto de vista nutricional, sería la materia seca, que determinaría la condición de consumo final de las especies en la dieta de los manatíes de la zona. U. mutica y $P$. repens fueron las especies mayoritariamente consumidas por los manatíes y ambas presentan valores de materia seca, que alcanzan casi el doble de lo encontrado para el resto de las especies consideradas (incluida E. crassipes).

La situación anterior podría explicarse según los planteamientos de la Teoría de Forrajeo Óptimo (Stephens y Krebs, 1986), donde aquellas plantas que proveen mayor cantidad de materia en cada ocasión de consumo podrían ser las que brindan la mayor cantidad de materia orgánica, lo cual optimizaría la energía requerida para alimentarse, si se compara con el resto de plantas que fueron evaluadas.

Desde un punto de vista nutricional, la selección de estos pastos, como las plantas de mayor relevancia en la dieta del manatí, podría deberse a varios factores:

1. Los manatíes poseen un tipo de sistema digestivo conocido como fermentación postgástrica, que se presenta en herbívoros no rumiantes como los caballos (Burn y Odell, 1987). Este tipo de digestión ocurre principalmente en la parte distal del intestino grueso (ciego y colon), lo cual promueve la ingesta de alimentos considerados de "baja calidad" (altos en contenido de fibra y bajos en contenido proteico), debido al recorrido que deben hacer los alimentos por el tracto digestivo (Rommel et al. n.d.; Smith, 1993; Launchbaugh et al. 2001), tal es el caso de los pastos, que tradicionalmente han constituido una parte importante en la dieta de los manatíes (O’Shea, 1994).

2. Además de las características citadas anteriormente, los manatíes poseen una serie de adaptaciones en su sistema digestivo (arrugas en el colon), que les permiten extraer más eficientemente la celulosa que otras especies con un sistema digestivo similar (Reynolds y 
Rommel, 1996). Esta condición de su sistema digestivo permite explicar, en parte, las bajas tasas metabólicas que presenta la especie (los alimentos tardan alrededor de 7 días, inclusive la literatura reporta hasta 14 días, en recorrer el tracto digestivo) (Rommel et al. n.d.; Reynolds y Rommel, 1996) y la obtención de ácidos grasos volátiles como fuente primordial energética para la especie, producto de la digestión de la celulosa (Burn y Odell, 1987).

3. El hecho de que la cantidad de materia seca contenida en las plantas analizadas esté definiendo el acomodo de estas en la dieta del manatí, podría deberse a una cuestión de forrajeo óptimo (Stephens y Krebs, 1986), aquellas plantas que aportan más alimento en cada bocado serían las más apetecidas.

La cantidad de lignina, como factor fundamental en la definición del valor nutricional de una especie (Van Soest, 1967), no parece estar definiendo con claridad la preferencia de los manatíes hacia el gamalote frente al pará, como especies mayoritariamente seleccionadas. A pesar de que el gamalote contiene los valores más altos de lignina, fue la especie preferida por los manatíes.

Otros factores que afectan el consumo podrían ser los que definan la preferencia de los manatíes por $P$. repens. Por ejemplo, la palatabilidad es un factor que afecta el consumo, en dependencia de variaciones propias de cada planta relacionadas con la apariencia, el olor, el sabor, la textura, entre otras (Church y Pond, 1988). P. repens es una planta más frágil y de mayor tamaño en estructuras como la hoja o el tallo, en relación con U. mutica. Esta situación podría definir la preferencia, ya que la cantidad consumida de $P$. repens en una misma área podría ser mayor que al consumir U. mutica.

La presente investigación ha permitido cuantificar la forma en que el manatí consume los recursos alimenticios en la zona. Esta ha sido la base para otras investigaciones que requieren de información sobre los elementos de la dieta para el cebado como técnica de captura para el posterior desarrollo de investigaciones con telemetría, lo que permitiría abordar vacíos sobre aspectos ecológicos fundamentales de la especie como en el área de estudio: sitios de distribución, patrones de migración y fidelidad de sitio, entre otros.

Otro aspecto relevante de la caracterización de la dieta desarrollada a través de este estudio fue la propuesta de conservación adecuada de áreas con dominancia de las plantas, como P. repens y U. mutica, identificadas como fundamentales en la dieta de los manatíes para la zona, ya que, por ejemplo, la distribución de $P$. repens está concentrada en pequeños sectores del PNT y uno de esos sectores se encuentra entre los límites del PNT y la comunidad de Tortuguero, lo que expone a las poblaciones de este pasto a múltiples factores antropogénicos.

\section{AGRADECIMIENTOS}

A la Fundación Rufford, de Inglaterra, por el financiamiento mayoritario del desarrollo de la etapa de campo de esta investigación; al Instituto Internacional en Conservación y Manejo de Vida Silvestre y a Jorge Fallas, por proporcionar aspectos logísticos y académicos para el desarrollo de esta investigación; al Área de Conservación Tortuguero, por el apoyo de estadía parcial en la etapa de toma de datos; al Hotel Manatus, a Fernando y Lili Figuls, por facilitar y fomentar la 
investigación de manatíes en Costa Rica; a Ignacio Jiménez Pérez, por las observaciones y los comentarios oportunos para la elaboración del presente documento; a Michael McCoy Bolton, por la disposición y motivación en el desarrollo de este trabajo; a James Powell, Robert Bonde y Lucy Keith, por dar forma a la idea de esta investigación; a Arilyn Pinnock, José Luis Alvarado, Heidy Saborío Salas y Daryl Loth, por su apoyo fundamental durante diferentes etapas de este trabajo; a la Fundación Salvemos al Manatí de Costa Rica, por el préstamo de equipo fundamental para el traslado en el área de estudio; a Dora Ingrid River Luther, por la colaboración en la identificación de plantas y al Laboratorio de Análisis de Productos Animales y Vegetales de la Universidad Nacional de Costa Rica, por las pruebas y la orientación brindadas para el análisis nutricional.

\section{BIBLIOGRAFÍA}

Altrichter, M. (1997). Estrategia de alimentación y comportamiento del chancho cariblanco (Tayassu pecari) en un bosque húmedo tropical de Costa Rica. Tesis de Maestría no publicada. Universidad Nacional de Costa Rica.

AOAC (Asociation of Official Analytical Chemists). (1984). Official Methods of Analisis. Edición 14. Washington, D.C., Estados Unidos: Editorial Santa Ana.

Aranda, M. (2000). Huellas y otros rastros de los mamíferos grandes y medianos de México. Xalapa, México: Instituto de Ecología, A. C.

Barrantes, J., Liao, A. \& Rosales, A. (1985). Atlas Climatológico de Costa Rica. Ministerio de Agricultura y Ganadería. Instituto Meteorológico Na- cional. San José, Costa Rica: Editorial Costa Rica.

Burn, D. (1986). The Digestive Strategy and Eficiency of the West Indian Manatee, Trichechus manatus. Biochem. Phys., 85A (1), 139-142.

Burn, D. \& Odell, D. (1987). Volatile Fatty Acid Concentrations in the Digestive Tract of the West Indian Manatee, Trichechus manatus. Biochem. Phys., $88 B$ (1), 47-49.

Byers, R. \& Steinhorst, R. (1984). Clarification of a Technique for Analisys of Utilization-Availability Data. J. Wildl. Manage., 48 (3), 1050-1053.

Church, D. C. \& Pond, W. G. (1988). Basic Animal Nutrition and Feeding. Third Edition. New York, EE. UU.: Published by John Wiley and Sons.

Colares, I. \& Colares, E. (2002). Food Plants Eaten by Amazonian Manatee (Trichechus inunguis, Mammalia: Sirenia). Braz. Arch. Biol. Tech., 45 (1), 67-72.

Guterres, M., Marmontel, M., Martins, D., Farias, R. \& Bustos, R. (2008). Anatomia e Morfologia de Plantas Aquáticas da Amazônia Utilizadas como Potencial Alimento por Peix-Boi Amazônico. São Pablo, Brasil: Instituto de Desenvolvimento Sustentável.

IMN (Instituto Meteorológico Nacional). (1975-1995). Datos de precipitación Área de Tortuguero. San José, Costa Rica: Gestión de Información y Comercialización.

IRET (Instituto Regional de Estudios en Sustancias Tóxicas). (2007). Análisis de agua y peces en un evento de contaminación dentro de diferentes cursos de agua en el Parque Nacional Tortuguero. Heredia, Costa Rica: Universidad Nacional de Costa Rica. 
Jiménez, I. (1998). Ecología y conservación del manatí antillano (Trichechus manatus) en el noreste de Costa Rica. Base de datos de los humedales del noreste de Costa Rica asociada a un sistema de información geográfica. Tesis de Maestría no publicada en Manejo y Conservación de Vida Silvestre. Universidad Nacional, Heredia, Costa Rica.

Jiménez, I. (1999). Estado de Conservación, Ecología y Conocimiento Popular del Manatí (Trichechus manatus) en Costa Rica. Vid. Silv. Neotrop., 8 (1-2), 18-30.

Jiménez, I. (2003). Los manaties del río San Juan y los Canales de Tortuguero: ecología y conservación. San José, Costa Rica: Amigos de la Tierra.

Johnson, D. (1980). The Comparison of Usage and Availability Measurements for Evaluating Resourses Preferente. Ecol., 61 (1), 65-71.

Launchbaugh, K., Provenza, F. \& Pfister, J. (2001). Herbivore Response to Anti-quality Factors in Forages. J. Range Manage., 54, 431-440.

Litvaitis, J., Titus, K. \& Anderson, E. (1994). Measuring Vertebrate Use of Terrestrial Habitats and Food. En Research and Management Techniques for Wildlife and Habitat (pp. 254274.). U.S. Fish and Wildlife Service. EE. UU.: Wildlife Society.

López, M., Altrichter, M., Sáenz, J. \& Eduarte, E. (2006). Valor Nutricional de los Alimentos de Tayassu pecari (Atiodactyla: Tayassuidae) en el Parque Nacional Corcovado, Costa Rica. Rev. Biol. Trop., 54 (2), 687-700.

Manly, B., McDonald, P., Thomas, D., MacDonald, T. \& Wallace, E. (2002). Resource Selection by Animals: Statistical Design and Analysis for Field
Studies. San Diego, CA, EE. UU.: Editorial Springer.

Manugistics, Statgraphics Centurium, XV. (2005). Launching the program, and creating a simple data file [Computer program manual]. Estados Unidos: StatPoint Technologies, Inc.

Mignucci-Giannoni, A. (1998). The Diet of tha Manatee (Trichechus manatus) in Puerto Rico. M. Mamm. Scien., 14 (2), 394-397.

O'Shea, T. (1994). Manatees. Washington, D.C., EE. UU.: Scientific American.

Provancha, J. \& Hall, C. (1991). Observations of Associtions Between Seagrass Beds and Manatee in East Central Florida. Florida Scientis, 54 (2), 87-98.

Reep, R. \& Bonde, R. (2006). The Florida Manatee: biology and conservation. Florida, EE. UU.: University Press of Florida.

Reynolds, J. \& Odell, D. (1991). Manatees and Dugongs. Miami, EE. UU.: Library of Congress Cataloging, Florida.

Reynolds, J. \& Rommel, S. (1996). Structure and Function of the Gastrointestinal Tract of the Florida Manatee, Trichechus manatus latirostris. Anat. Rec., 245, 539-558.

Reynolds, J., Szelistowski, W. \& León, M. (1995). Status and Conservation of Manatee Trichechus manatus manatus in Costa Rica. Biol. Conserv., 71, 193-196. Rommel, S., Reynolds, J. \& Lynch, H. (n.d.). Adaptations of Herbivorous Marines Mammals. North Carolina State University College of Veterinary Medicine. Florida, Estados Unidos: U.S. Fish and Wildlife Service.

Smethurst, D. \& Nietschmann, B. (1999). The Distribution of Manatees (Trichechus manatus) in the Coastal Wa- 
terways of Tortuguero, Costa Rica. Thomas, D., Manly, B. \& Mc Donald, L. Biol. Conserv., 89, 267-274.

Smith, K. (1993). Manatee Habitat and Human-related Threats to Seagrass in Florida: A review. Florida, EE. UU.: Departamento de Protección al Medio Ambiente.

Sokal, R. \& Rohlf, J. (1979). Biometría: Principios y métodos estadísticos en la investigación biológica. Madrid, España: Ediciones H. Blume.

Stephens, D. \& Krebs, J. (1986). Foraging Theory. Nueva York, Estados Unidos: Universidad de Princeton. (2001). A Unified Theory for the Study of Resource Selection (Availability and Use) by Wildlife Populations. En Populations (pp. 56-64), D. $\mathrm{McCu}-$ lloungh y R. Barrett, (Eds.), Inglaterra: British Library.

Van Soest, P. (1967). Development of a Comprehensive System of Feed Analysis and its Application to Forages. J. Anim. Sci., 26, 119-128. 